\title{
Treatment of Patella Infera Following Closed Trauma by a Combination of Patella Tendon Tenotomy and Tuberositas Tibia Osteotomy and Augmentation with Semitendinosus Tendon Autograft
}

\author{
Jacob Schade Engbjerg ${ }^{1}$, Philippe Nicolini ${ }^{1}$ and Rikke Thorninger ${ }^{1 *}$ \\ Department of Orthopaedics, Regionshospitalet Randers, Randers, Denmark. \\ ${ }^{\star}$ Corresponding author: BRikke Thorninger, Department of Orthopaedics, Regionshospitalet Randers, Randers, Denmark, Email: rikkthor@rm.dk
}

Received: April 24, 2020; Accepted: May 05, 2020; Published: May 11, 2020

Keywords: Patella infera, patella baja, patella tendon tenotomy, Z-plasty, tuberositas tibia osteotomy, tendon autograft augmentation, semitendinosus.

\begin{abstract}
Abbreviations: $\mathrm{ROM}=$ Range of Motion
\section{Introduction}

Patella Infera (PI) is a rare condition presenting with shortening of the patella tendon. PI can be seen as a congenital abnormality, or as a complication secondary to trauma or kneesurgery [1-5]. It was first described in 1982 by Caton et al. and was defined as a CatonDeschamps index $£ 0.66$. The Caton-Deschamps index is assessed on a knee $\mathrm{x}$-ray in sagittal view where the length of the patella tendon, defined as the distance from the lower patella pole to the superior part of the tibial tubercle, is divided by the length of the patella, defined as the greatest diagonal length measured [6]. A normal Caton-Deschamps index is defined as an index in the range $>0.6$ or $<1.26$. Clinically the condition presents with symptoms of decreased ROM, lach of knee flexion, patellofemoral knee pain and accelerated progression of patellofemoral osteoarthritis [3].
\end{abstract}

There is no consensus on treatment method of patella infera, it has been proposed that surgery is indicated, when the Caton-Deschamps index is lower than or equals 0.6 (6). The two main surgical treatment modalities include a z-plasty of the patella tendon or an osteotomy of the tibial tuberositas. It has been suggested to use a z-plasty, when the Caton-Deschamps index is $\leq 0.6$ and the patella tendon is $<25 \mathrm{~mm}$, and to use the tuberositas osteotomy when the CatonDeschamps index is $\leq 0.6$ and the patella tendon is $>25 \mathrm{~mm}$ (6). Immediate postoperative mobilization and physiotherapy is required to learn to activate the quadriceps muscle, and focus on strengthening exercises of the muscle has been described to be the most important post-operative regime to prevent relapse of patella infera $[5,6]$. This case describes a severe secondary developed patella infera after conservative treatment of a patella fracture. Surgical technic is done with a combination of patella tendon tenotomy and tuberositas tibia osteotomi and augmentation with semitendinosus allograft. Previous reports of surgical treatment of patella infera is done with either a z-plasty or tuberositas tibia osteotomy, no previous reports have to our knowledge described the surgical treatment of patella infera with a combination of the two treatment modalities.

\section{Case Report}

A healthy fifty-five year old woman slipped and fell onto her left knee while walking. She reported pain and difficulties in both flexion and extension of the knee.X-ray revealed a comminuted non-displaced fracture of the inferior pol of the patella (Figure 1). The knee was immobilized in full extension for 6 weeks and weight-bearing was allowed within the limits of pain. X-ray made 3 months after the injury revealed early union of the fracture. Five months following the fracture she presented with limited range of motion with reduced knee-flexion to 95 degrees despite a specialized physiotherapy rehabilitation training program. The rehabilitation program was continued but 8 months following the fracture she presented with pain, aggravation of the affected knee flexion and inability to follow her rehabilitation. Clinical examination revealed atrophy of the quadriceps muscle, knee-flexion reduced to 30 degrees, full knee-extension but inability to raise the leg in full extension. A MR-scan and $\mathrm{x}$-ray showed severe patella infera with a Caton-Deschamps index of 0.3 and a patella tendon measuring only $13 \mathrm{~mm}$ (Figure $2 \mathrm{a}+$ Figure $2 \mathrm{~b}$ ).

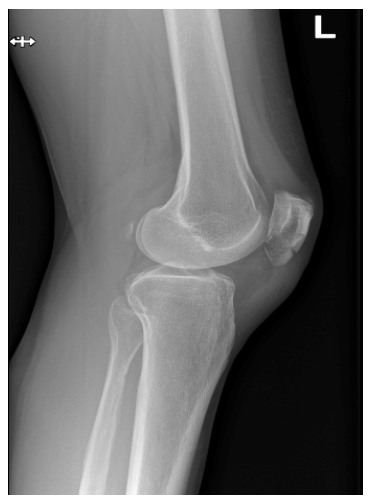

Figure 1: X-ray showing a comminute non-displaced fracture of the inferior patella pol of after closed trauma to the left knee. 
Rikke Thorninger (2020) Treatment of patella infera following closed trauma by a combination of patella tendon tenotomy and tuberositas tibia osteotomy and augmentation with semitendinosus tendon autograft.
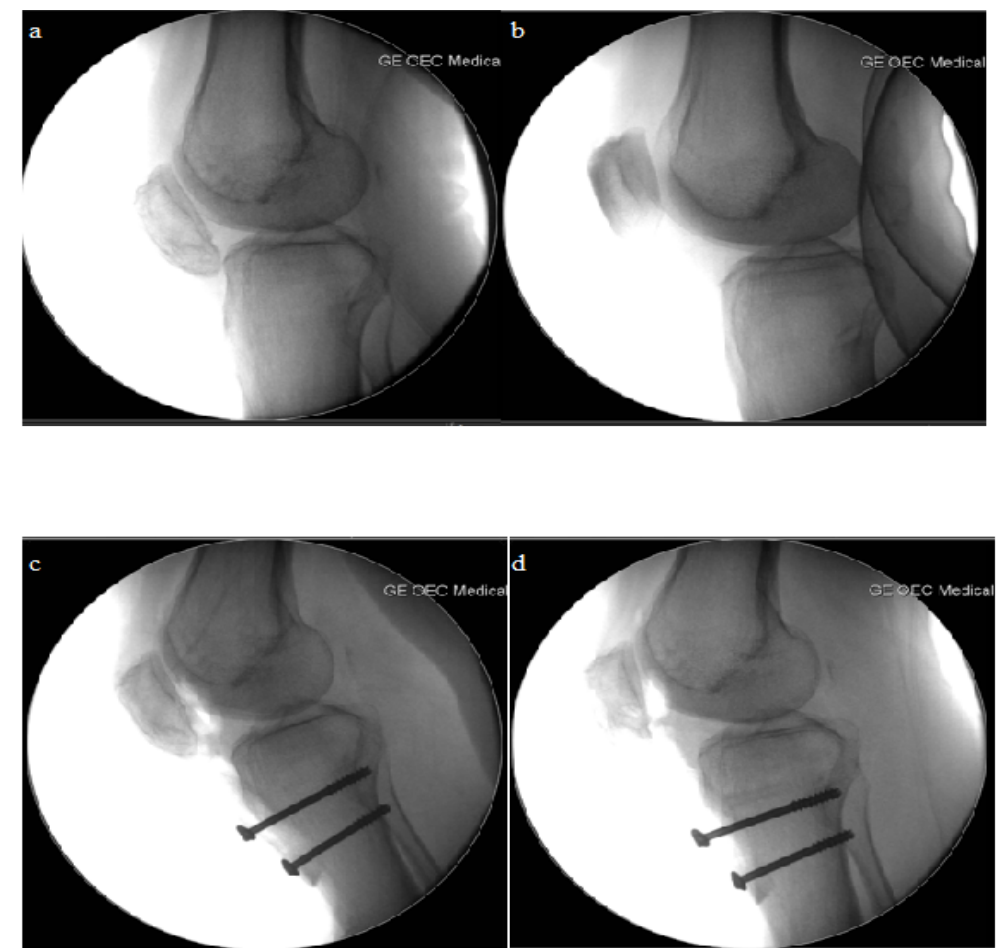

Figure 2: A: Pre-operative $\mathrm{x}$-ray of the left knee with distinct patella-infera with a Caton-index of $0.3 \mathrm{~B}$ : Pre-operative $\mathrm{x}$-ray of the right healthy knee, no patella infera. C: X-ray of the left knee after osteotomy, Caton index $=0.56 . \mathrm{D}$ : X-ray after osteotomy and Z-plasty with a Caton-Deschamps index of 0.8 as a final result.
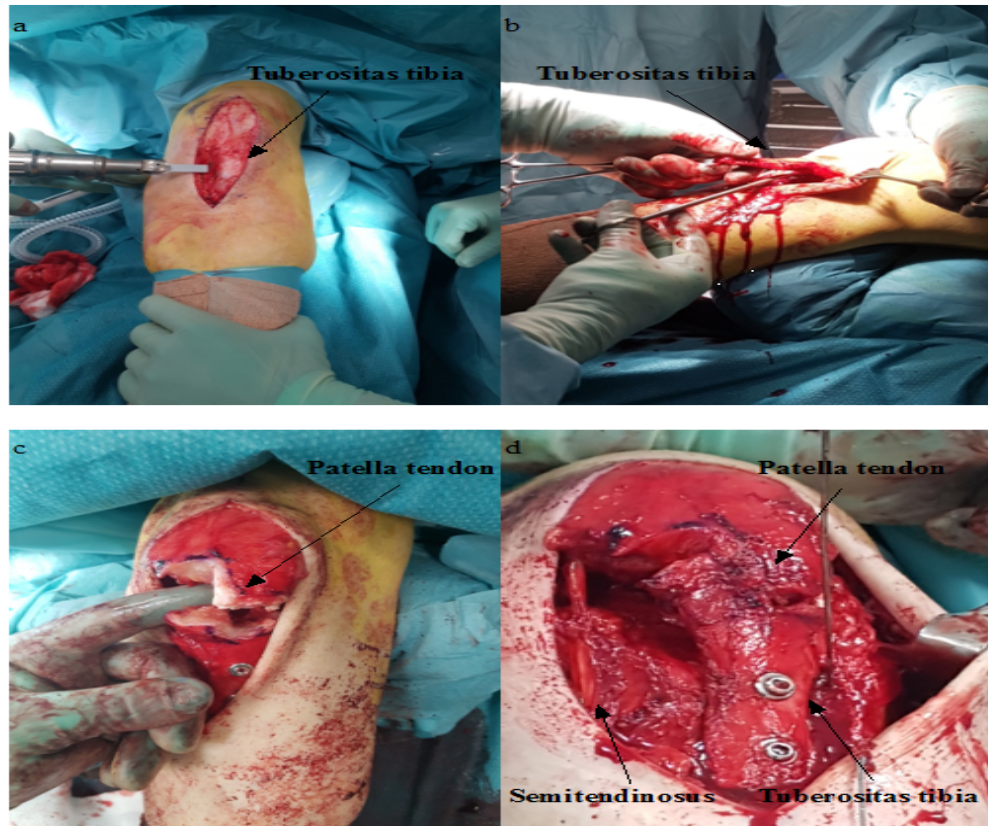

Figure 3: A and B shows the osteotomy of the tuberositas tibia. C: Illustrates the z-plasty of the patella-tendon, note the markedly sclerosis of the tendon. D: Final result after osteotomy, z-plasty and augmentation with the semitendinosus-tendon.

The Caton-Deschamps index was at time of injury 0,9 as well as 9 days later. After 45 days it reduced to 0,44 . After 10 months the measurement was 0,3 reduced to 0,2 after one year. Because of the severity of the patella infera it was planned to preform both a osteotomy of the tibial tuberositas and a z-plasty of the patella-tendon following augmentation with the semitendinosus-tendon. Pre-operative an Oxford 12-item Knee Score was made resulting in a score of 7 .

\section{Results}

First, a osteotomy was preformed (Figure $3 a+$ Figure $3 b$ ) and the tibial tuberositas was inserted $18 \mathrm{~mm}$ proximal to the origin resulting in a Caton-index of 0.56 ( 0.25 prior) (Figure $2 \mathrm{c})$. Thereafter, the patella-tendon was extended by $1 \mathrm{~cm}$ to a full length of $23 \mathrm{~mm}(13 \mathrm{~mm}$ prior) by a z-plasty (Figure $3 \mathrm{c}$ ) resulting in a final Caton-Deschamps index of 0.8 (Figure 2d). Finally the tendon was augmented by the 
semitendinosus-tendon (Figure 3d). After the procedure it was possible for the operator to flex the knee 110 degree (30 degree pre-operative). Post-operative regime was planned which included 1) mobilization with don-joy-bandage in 0-30 degree and only partial weight bearing in full extension for 2 weeks, then 0-60 degrees for 2 weeks and full weight-bearing and then 0-90 degrees for 2 weeks, 2) CMP-machine with mobilization 0-60 degrees without the bandage 4 times per day for 4 weeks, 3) early training instructed by physiotherapist and 4) ELstimulation of the quadriceps muscle. 8 weeks after the operation she was able to perform knee flexion to 95 degrees and had a good knee stability and she was given no limitation in range of motion. 3 months after the operation she was capable of performing 100 degrees knee flexion and full extension at clinical examination. The Oxford 12-item Knee Score was repeated resulting in a score of 42 which indicates satisfactory joint function.

\section{Discussion}

This case report describes a sucessefull treatment of PI with a combination of two well established surgical methods; a tibial tubercle transfer and a Z-plasty of the patella tendon.Because of the poor quality of patella tendon after post traumatic patella infera it was decided to reinforces it with the semitendinosus-tendon. It assures a safe heeling of the Z-plasty and allows a postoperative early mobilization by ROM as well as an aggressive rehabilitation that is in this case extremely important to get a good final result.
The literature has no clear consensus on optimal treatment of PI and the rarity of PI does not allow any RCT study to be performed. To our knowledge it is the first time the two surgical approaches has been combined, and the obtained Caton-Dechampes index and high Oxford knee score confirms our theory of a succesfull combination. However our weakness could be the short follow up period mainly do to relapse as previous described in the literature. In conclusion the combined surgical methods could be an alternative in treating patella infera.

\section{References}

1. Morshed S, Ries MD (2002) Patella infera after nonoperative treatment of a patellar fracture: A case report. J Bone Joint. Surg Am 84: 1018-1021. [Crossref]

2. Jiang X, Zhang YM, Liu JY (2013) Patella infera following patellar tendon contracture after closed trauma. Chin Med J (Engl) 126: 3990-3991. [Crossref]

3. Kennedy MI, Aman Z, DePhillipo NN, LaPrade RF (2019) Patellar tendon tenotomy for treatment of patella baja and extension deficiency. Arthrosc Tech 8: 317-320.

4. Guido W, Christian H, Elmar H, Elisabeth A, Christian F (2016) Treatment of patella baja by a modified Z-plasty. Knee Surgery, Sport Traumatol Arthrosc 24: 2943-2947. [Crossref]

5. Bruhin VF, Preiss S, Salzmann GM, Harder LP (2016) Frontal tendon lengthening plasty for treatment of structural patella baja. Arthrosc Tech 5: $1395-1400$.

6. Caton JH (2010) The management of patella infera in current practice. Eur J Orthop Surg Tramatol 20: 265-271.

\section{Citation:}

Rikke Thorninger (2020) Treatment of patella infera following closed trauma by a combination of patella tendon tenotomy and tuberositas tibia osteotomy and augmentation with semitendinosus tendon autograft. Integr J Orthop Traumatol Volume 3(1): 1-3. 